\title{
Laplace transform - finite element method for non Fickian flows
}

\author{
S. Barbeiro ${ }^{\text {a }}$, S.Gh. Bardeji ${ }^{\mathrm{a}}$, J.A. Ferreira ${ }^{\mathrm{a}}$
}

${ }^{a}$ CMUC, Department of Mathematics, University of Coimbra, Apartado 3008, EC Universidade, 3001-454 Coimbra, Portugal

\begin{abstract}
In this paper we consider numerical methods for integro-differential problems based on time discretization via Laplace transformation. We focus our attention in models arising in the context of non Fickian solute transport phenomena in porous media. The mathematical models which describe the evolution of the solute concentrations are characterized by Volterra equations. We present and analyze an hybrid method which combines the Laplace transformation with respect to the time variable with the finite element discretization in the spatial variables. Numerical results illustrate the performance of the method.
\end{abstract}

Keywords: Integro-differential equation, Laplace transformation, finite elements.

\section{Introduction}

In this paper we consider the following Volterra equation

$$
\frac{\partial c}{\partial t}(t)+A c(t)+\int_{0}^{t} k(t-s) B c(s) d s=f(t), t>0
$$

with

$$
\begin{aligned}
& A c(t)=-\nabla \cdot\left(A_{22} \nabla c(t)\right)+\nabla \cdot\left(A_{2} c(t)\right)+A_{1} c(t), \\
& B c(t)=-\nabla \cdot\left(B_{22} \nabla c(t)\right)+\nabla \cdot\left(B_{2} c(t)\right)+B_{1} c(t),
\end{aligned}
$$

where $k$ denotes the kernel, and $A_{22}, B_{22}, A_{2}, B_{2}, A_{1}$ and $B_{1}$ represent functions dependent on $(x, y)$, being $A_{22}=\left[a_{i j}\right]$ and $B_{22}=\left[b_{i j}\right] 2$ by 2 symmetric matrix functions, $A_{2}=\left[a_{i}\right]$ and $B_{2}=\left[b_{i}\right]$ vectorial functions and $A_{1}$ and $B_{1}$ scalar functions.

Solute transports in porous media are commonly characterized by the convectiondiffusion equation

$$
\frac{\partial c}{\partial t}+\nabla \cdot(v c)=\nabla \cdot(D \nabla c)+f \text { in } \Omega \times(0, T],
$$

Email addresses: silvia@mat.uc.pt (S. Barbeiro), gholamii_somaye@yahoo.com (S.Gh. Bardeji), ferreira@mat.uc.pt (J.A. Ferreira)

URL: http://www.mat.uc.pt/ silvia (S. Barbeiro), http://www.mat.uc.pt/ f ferreira (J.A. Ferreira) 
where $c$ denotes the solute concentration, $D$ denotes the dispersion tensor (which can be $c$ dependent) and $v$ represents the fluid velocity. $\Omega$ is the spatial domain with boundary $\partial \Omega$. Equation (2) is established using the so called Fick's law for the mass flux due to molecular diffusion, $J_{d}$, and the convective flux, $J_{c}$, given by, respectively,

$$
J_{d}=-D \nabla c
$$

and

$$
J_{c}=v c .
$$

Then the mass flux $J=J_{d}+J_{c}$ is given by

$$
J=v c-D \nabla c,
$$

which combined with the mass conservation equation

$$
\frac{\partial c}{\partial t}+\nabla \cdot J=f
$$

leads to the convection-diffusion equation (2).

When the memory effect of the fluid flow has an important role in the solute transport, equation (2) should be modified in order to incorporate such effect. One possible approach to accomplish this is to assume that the mass flux $J_{d}$ is given by

$$
J_{d}(t)=-\frac{1}{\tau} \int_{0}^{t} e^{-\frac{t-s}{\tau}} D \nabla c(s) d s+J_{d}(0),
$$

where $\tau$ is a relaxation parameter. Combining now $J=J_{c}+J_{d}$ with $J_{d}$ given by (7) with the mass conservation equation (6) we arrive to

$$
\frac{\partial c}{\partial t}+\nabla \cdot(v c)=\frac{1}{\tau} \int_{0}^{t} e^{-\frac{t-s}{\tau}} \nabla \cdot(D \nabla c(s)) d s+f-\nabla J_{d}(0) \text { in } \Omega \times(0, T],
$$

that replaces (2) for non Fickian flows.

Equation (8) is a particular case of an equation of type (1), which is the model that we are going to study in this article. This type of equations have been proposed in the literature to describe non Fickian diffusion processes as for instance in [11], [23], [24], [27], [29]. The development of efficient and accuracy numerical methods to solve the initial boundary value problem (IBVP) defined by (1) has attracted the attention of several researchers during the last two decades. A significative number of contributions can be found in the literature. Without be exhaustive we mention [25], [26], [41], [44] for the study of finite element semi-discrete approximations, [32] for the study of semi-discrete lumped mass approximations, [16], [17] and [35] for the study of finite volume semi-discrete approximations, [2], [4], [5], [6], [8], [18], [20] and [21] for finite difference methods presenting the same qualitative behavior of the integro-differential problem.

Integro-differential equations (1) can be rewritten as equivalent linear differential systems: a partial differential equation involving only a time derivative and an integro-differential equation presenting only partial derivatives with respect to the space variables. This approach was used, for instance, in [18] and recently in [36] where mixed finite element methods were used for the disretization. Systems of differential equations that are equivalent to nonlinear versions of equation (1) were considered in [7] and [33]. 
In what follows we will consider that $\Omega \subset \mathbb{R}^{2}$ is bounded polygonal domain. We will introduce an hybrid method for the IBVP defined by (1), with the Dirichlet boundary condition

$$
c(t)=\psi(t) \text { on } \partial \Omega \times \mathbb{R}^{+},
$$

and with the initial condition

$$
c(0)=c_{0} \text { in } \Omega \text {. }
$$

The method is based on the use of Laplace transform to the IBVP $(1),(9),(10)$ which converts the IBVP in an elliptic boundary value problem that depends on the Laplace parameter. The elliptic problem is solved by using finite element methods for the spatial variables, for a choice of a finite set of quadrature points in the Laplace domain. This set of elliptic equations can be solved in parallel. Finally the numerical approximation for the solution on the physical time space domain is obtained by using numerical inverse Laplace transforms. The use of Laplace transforms to solve nonlocal flow and transport equations has been discussed in the literature, as for instance in [22], [39], [40] and [43],. This type of approach was also considered in [3], [15], [19], [34], [37], [38]. The convergence analysis of methods designed using this procedure were presented e.g. in [9], [28] and [42] (see also the references cited in these two last papers). The present paper presents error bounds with respect $H^{1}$-norm which are based on the Paley-Wiener Theorem and the generalization of the classical arguments of the finite element analysis to complex Sobolev spaces ([9]). This type of approach allow us to consider more general differential and integro-differential operators when compared with those studied in [28].

The paper is organized as follows. In Section 2 we introduce the variational formulation of the IBVP (1), (9), (10) and its finite element formulation. The weak variational problem in the Laplace space is introduced in Section 3 and the existence and uniqueness of the solution of this problem are also studied in this section. In Section 4 we describe the finite element approximation of the variational problem introduced in the previous section and an error estimate for such approximation is established. Using the Paley-Wiener Theorem we return to the initial variables and we estimate the error for Laplace inverse of the finite element solution in the Laplace space. Finally some numerical experiments illustrating the convergence results are also included.

\section{Weak solution and its Ritz-Galerkin approximation}

Let $L^{2}(\Omega), H^{1}(\Omega)$ be the usual Sobolev spaces endowed, respectively, with the usual inner products $(.,),.(., .)_{H^{1}(\Omega)}$ and norms $\|\cdot\|_{L^{2}(\Omega)},\|\cdot\|_{H^{1}(\Omega)}$. The space of functions $v \in H^{1}(\Omega)$ such that $v=0$ on $\partial \Omega$, is denoted by $H_{0}^{1}(\Omega)$. By $L^{2}\left(\mathbb{R}^{+}, H^{1}(\Omega)\right)$ we denote the space of functions $v: \mathbb{R}^{+} \rightarrow H^{1}(\Omega)$ such that

$$
\int_{\mathbb{R}^{+}}\|v(s)\|_{H^{1}(\Omega)}^{2} d s<\infty
$$

and by $H^{1}\left(\mathbb{R}^{+}, L^{2}(\Omega)\right)$ we denote the space of functions $v: \mathbb{R}^{+} \rightarrow H^{1}(\Omega)$ such that

$$
\int_{\mathbb{R}^{+}}\left(\|v(s)\|_{L^{2}(\Omega)}^{2}+\left\|\frac{d v}{d t}(s)\right\|_{L^{2}(\Omega)}^{2}\right) d s<\infty .
$$


In (12), the time derivative is in the weak sense.

The weak solution for the IBVP (1), (9), (10) is obtained solving the following problem: find $c \in L^{2}\left(\mathbb{R}^{+}, H^{1}(\Omega)\right) \cap H^{1}\left(\mathbb{R}^{+}, L^{2}(\Omega)\right)$ such that $c(t)=\psi(t)$ on $\partial \Omega$ and, for any $T>0$,

$$
\left\{\begin{aligned}
\left(\frac{\partial c}{\partial t}(t), v\right) & +\left(\left(A_{22} \nabla c(t), \nabla v\right)\right)-\left(\left(c(t) A_{2}, \nabla v\right)\right)+\left(A_{1} c(t), v\right) \\
& +\int_{0}^{t} k(t-s)\left(\left(\left(B_{22} \nabla c(s), \nabla v\right)\right)-\left(\left(B_{2} c(s), \nabla v\right)\right)\right. \\
& \left.+\left(B_{1} c(s), v\right)\right) d s=(f(t), v) \text { a.e. in }(0, T), \forall v \in H_{0}^{1}(\Omega), \\
c(0)=c_{0} &
\end{aligned}\right.
$$

We remark that we use the notation

$$
((u, v))=\sum_{i=1}^{2}\left(u_{i}, v_{i}\right)
$$

for $u=\left(u_{1}, u_{2}\right), v=\left(v_{1}, v_{2}\right), u_{i}, v_{i} \in L^{2}(\Omega), i=1,2$.

To compute the the semi-discrete Ritz-Galerkin approximation $c_{H}$ for the weak solution $c$ defined by (13), we introduce in $\bar{\Omega}$ an admissible triangulation $\mathcal{T}_{H}$ and the corresponding finite dimension space

$$
V_{H, m}=\left\{u \in C^{0}(\bar{\Omega}): u(x)=P_{m}(x), x \in \Delta, \Delta \in \mathcal{T}_{H}\right\},
$$

where $P_{m}(x)$ denotes a polynomial in space variables with degree $\leq m$.

Then, given $c_{0, H} \in V_{H, m}, c_{H}$ is obtained solving the following problem: find $c_{H} \in L^{2}\left(\mathbb{R}^{+}, H_{0}^{1}(\Omega)\right) \cap H^{1}\left(\mathbb{R}^{+}, L^{2}(\Omega)\right)$ such that $c_{H}(t)=\psi(t)$ on $\partial \Omega$ and, for any $T>0$,

$$
\left\{\begin{aligned}
\left(\frac{\partial c_{H}}{\partial t}(t), v_{H}\right)+\left(\left(A_{22} \nabla c_{H}(t), \nabla v_{H}\right)\right)-\left(\left(c_{H}(t) A_{2}, \nabla v_{H}\right)\right)+\left(A_{1} c_{H}(t), v_{H}\right) \\
+\int_{0}^{t} k(t-s)\left(\left(\left(B_{22} \nabla c_{H}(s), \nabla v_{H}\right)\right)-\left(\left(c_{H}(s) B_{2}, \nabla v_{H}\right)\right)\right. \\
\left.+\left(B_{1} c_{H}(s), v_{H}\right)\right) d s=\left(f(t), v_{H}\right) \text { a.e. in }(0, T), \forall v_{H} \in V_{H, m}, \\
c_{H}(0)=c_{0, H}
\end{aligned}\right.
$$

In what follows we present an approach that allows us to compute an approximation for the weak solution of the IBVP (1), (9), (10) avoiding the computation of the solution of the integro-differential problem (14). We will also derive error estimates for the numerical solution.

\section{Weak solution in Laplace space}

In what follows we replace the IBVP (1), (9), (10) by the corresponding problem obtained applying the Laplace transform $\mathcal{L}$.

Applying Laplace transform to (1) we obtain

$$
\left(I_{d}+\frac{1}{p} A+\frac{\tilde{k}}{p} B\right) \tilde{c}=\frac{1}{p}\left(c_{0}+\tilde{f}\right) \text { in } \Omega,
$$


where $I_{d}$ is the identity operator, $\tilde{k}, \tilde{f}$ denote the Laplace transforms of $k$ and $f$, respectively, and $\tilde{c}$ is the Laplace transform of $c$. Equation (15) is complemented with the boundary condition

$$
\tilde{c}=\tilde{\psi} \text { on } \partial \Omega,
$$

where $\tilde{\psi}$ represents the Laplace transform of $\psi$.

In order to define the weak solution for the boundary value problem (15), (16) we introduce now the set of functional spaces need to this definition. We denoted by $\operatorname{Re} z$ the real part of $z \in \mathbb{C}$. Let $H^{1}\left(\Omega, \mathbb{C}_{\sigma}^{+}\right)$and $L^{2}\left(\Omega, \mathbb{C}_{\sigma}^{+}\right)$be the Sobolev spaces of functions that depend on the complex number $p \in \mathbb{C}_{\sigma}^{+}=\{p \in$ $\mathbb{C}: \operatorname{Re} p \geq \sigma>0\}$ where they are analytic. In $L^{2}\left(\Omega, \mathbb{C}_{\sigma}^{+}\right)$we consider the inner product

$$
(\tilde{u}, \tilde{v})=\int_{\Omega} \tilde{u} \tilde{v} d x, \tilde{u}, \tilde{v} \in L^{2}\left(\Omega, \mathbb{C}_{\sigma}^{+}\right),
$$

and the corresponding norm

$$
\|\tilde{u}\|_{L^{2}\left(\Omega, \mathbb{C}_{\sigma}^{+}\right)}=(\tilde{u}, \tilde{u})^{1 / 2}, \tilde{u} \in L^{2}\left(\Omega, \mathbb{C}_{\sigma}^{+}\right) .
$$

The inner product (17) allows us to introduce in $L^{2}\left(\Omega, \mathbb{C}_{\sigma}^{+}\right) \times L^{2}\left(\Omega, \mathbb{C}_{\sigma}^{+}\right)$the following inner product

$$
\left(\left(\left(\tilde{u}_{1}, \tilde{u}_{2}\right),\left(\tilde{v}_{1}, \tilde{v}_{2}\right)\right)\right)=\sum_{i=1}^{2}\left(\tilde{u}_{i}, \tilde{v}_{i}\right),\left(\tilde{u}_{1}, \tilde{u}_{2}\right),\left(\tilde{v}_{1}, \tilde{v}_{2}\right) \in L^{2}\left(\Omega, \mathbb{C}_{\sigma}^{+}\right) \times L^{2}\left(\Omega, \mathbb{C}_{\sigma}^{+}\right) .
$$

The space $H^{1}\left(\Omega, \mathbb{C}_{\sigma}^{+}\right)$is endowed with the inner product

$$
(\tilde{u}, \tilde{v})_{H^{1}\left(\Omega, \mathbb{C}_{\sigma}^{+}\right)}=(\tilde{u}, \tilde{v})+((\nabla \tilde{u}, \nabla \tilde{v})), \tilde{u}, \tilde{v} \in H^{1}\left(\Omega, \mathbb{C}_{\sigma}^{+}\right),
$$

which induces the following norm

$$
\|\tilde{u}\|_{H^{1}\left(\Omega, \mathbb{C}_{\sigma}^{+}\right)}=(\tilde{u}, \tilde{u})_{H^{1}\left(\Omega, \mathbb{C}_{\sigma}^{+}\right)}^{1 / 2}, \tilde{u} \in H^{1}\left(\Omega, \mathbb{C}_{\sigma}^{+}\right) .
$$

By $|\cdot|_{H^{1}\left(\Omega, \mathbb{C}_{\sigma}^{+}\right)}$we denote the following semi-norm in $H^{1}\left(\Omega, \mathbb{C}_{\sigma}^{+}\right)$

$$
|\tilde{u}|_{H^{1}\left(\Omega, \mathbb{C}_{\sigma}^{+}\right)}=((\nabla \tilde{u}, \nabla \tilde{u}))^{1 / 2}, \tilde{u} \in H^{1}\left(\Omega, \mathbb{C}_{\sigma}^{+}\right) .
$$

The subspace of $H^{1}\left(\Omega, \mathbb{C}_{\sigma}^{+}\right)$composed by the functions vanishing on $\partial \Omega$ is represented by $H_{0}^{1}\left(\Omega, \mathbb{C}_{\sigma}^{+}\right)$.

Let $a_{p}(.,):. H^{1}\left(\Omega, \mathbb{C}_{\sigma}^{+}\right) \times H^{1}\left(\Omega, \mathbb{C}_{\sigma}^{+}\right) \rightarrow \mathbb{C}$ be the sesquilinear form

$$
a_{p}(\tilde{u}, \tilde{v})=(\tilde{u}, \tilde{v})+\frac{1}{p}(a(\tilde{u}, \tilde{v})+\tilde{k} b(\tilde{u}, \tilde{v}))
$$

where

$$
a(\tilde{u}, \tilde{v})=\left(\left(A_{22} \nabla \tilde{u}, \nabla \tilde{v}\right)\right)-\left(\left(A_{2} \tilde{u}, \nabla \tilde{v}\right)\right)+\left(A_{1} \tilde{u}, \tilde{v}\right)
$$

and

$$
b(\tilde{u}, \tilde{v})=\left(\left(B_{22} \nabla \tilde{u}, \nabla \tilde{v}\right)\right)-\left(\left(B_{2} \tilde{u}, \nabla \tilde{v}\right)\right)+\left(B_{1} \tilde{u}, \tilde{v}\right)
$$

for $\tilde{u}, \tilde{v} \in H^{1}\left(\Omega, \mathbb{C}_{\sigma}^{+}\right)$. By $\ell: H^{1}\left(\Omega, \mathbb{C}_{\sigma}^{+}\right) \rightarrow \mathbb{C}$ we denote the following functional

$$
\ell(\tilde{v})=\frac{1}{p}\left(c_{0}+\tilde{f}, \tilde{v}\right)
$$


We associate with the sesquilinear form $a_{p}(.,$.$) the following operator L$ : $H_{0}^{1}\left(\Omega, \mathbb{C}_{\sigma}^{+}\right) \rightarrow H_{0}^{1}\left(\Omega, \mathbb{C}_{\sigma}^{+}\right)^{\prime}$

$$
L \tilde{u}(\tilde{v})=a_{p}(\tilde{u}, \tilde{v}),
$$

where $H_{0}^{1}\left(\Omega, \mathbb{C}_{\sigma}^{+}\right)^{\prime}$ denotes the dual space of $H_{0}^{1}\left(\Omega, \mathbb{C}_{\sigma}^{+}\right)$.

The existence and uniqueness of the solution of the variational problem: find $\tilde{c} \in H^{1}\left(\Omega, \mathbb{C}_{\sigma}^{+}\right)$such that $\tilde{c}=\tilde{\psi}$ on $\partial \Omega$ and

$$
a_{p}(\tilde{c}, \tilde{v})=\ell(\tilde{v}), \forall \tilde{v} \in H_{0}^{1}\left(\Omega, \mathbb{C}_{\sigma}^{+}\right),
$$

is established in the next result. To simplify the proof we consider homogeneous boundary conditions.

Theorem 1. Let $f \in L^{2}\left(\mathbb{R}^{+}, L^{2}(\Omega)\right), c_{0} \in L^{2}(\Omega)$ and $a_{i j}, b_{i, j}, a_{i}, b_{i}, A_{1}, B_{1} \in$ $L^{\infty}(\Omega), i, j=1,2$. If there exists $\sigma \in \mathbb{R}^{+}$and $e: \mathbb{C}_{\sigma}^{+} \rightarrow \mathbb{R}^{+}$such that, for $p \in \mathbb{C}_{\sigma}^{+}$, holds the following

$$
\operatorname{Re} a_{p}(\tilde{u}, \tilde{u}) \geq e(p)\|\tilde{u}\|_{H^{1}\left(\Omega, \mathbb{C}_{\sigma}^{+}\right)}^{2}, \forall \tilde{u} \in H_{0}^{1}\left(\Omega, \mathbb{C}_{\sigma}^{+}\right),
$$

then the variational problem (24) with $\psi=0$ has only one solution $\tilde{c} \in H_{0}^{1}\left(\Omega, \mathbb{C}_{\sigma}^{+}\right)$.

Proof: In the proof of this result we use the Lax-Milgram Theorem. We start by noticing that if $f \in L^{2}\left(\mathbb{R}^{+}, L^{2}(\Omega)\right), c_{0} \in L^{2}(\Omega)$ then the linear functional $\ell: H^{1}\left(\Omega, \mathbb{C}_{\sigma}^{+}\right) \rightarrow \mathbb{C}$ defined by $(23)$ belongs to $H_{0}^{1}\left(\Omega, \mathbb{C}_{\sigma}^{+}\right)^{\prime}$. In what follows we prove that the sesquilinear form $a_{p}(.,):. H_{0}^{1}\left(\Omega, \mathbb{C}_{\sigma}^{+}\right) \times H_{0}^{1}\left(\Omega, \mathbb{C}_{\sigma}^{+}\right) \rightarrow \mathbb{C}$ defined by (20) is elliptic, that is, there exist positive constants $a_{c, p}$ and $a_{e, p}$ such that

$$
\left|a_{p}(\tilde{u}, \tilde{v})\right| \leq a_{c, p}\|\tilde{u}\|_{H^{1}\left(\Omega, \mathbb{C}_{\sigma}^{+}\right)}\|\tilde{v}\|_{H^{1}\left(\Omega, \mathbb{C}_{\sigma}^{+}\right)}, \quad \forall \tilde{u}, \tilde{v} \in H_{0}^{1}\left(\Omega, \mathbb{C}_{\sigma}^{+}\right),
$$

and

$$
\left|a_{p}(\tilde{u}, \tilde{u})\right| \geq a_{e, p}\|\tilde{u}\|_{H^{1}\left(\Omega, \mathbb{C}_{\sigma}^{+}\right)}^{2}, \quad \forall \tilde{u} \in H_{0}^{1}\left(\Omega, \mathbb{C}_{\sigma}^{+}\right) .
$$

To establish (26) we need only to use the fact that the coefficients functions are assumed to be in $L^{\infty}(\Omega)$. The condition $(27)$ is a trivial consequence of the assumption (25).

In the next results we specify necessary conditions that guarantee that the assumption (25) holds true. We start associating with $a(.,$.$) defined by (21)$ the following sesquilinear forms

$$
\begin{gathered}
a_{I}(\tilde{u}, \tilde{v})=\left(\left(A_{22} \nabla \tilde{u}, \nabla \tilde{v}\right)\right), \tilde{u}, \tilde{v} \in H^{1}\left(\Omega, \mathbb{C}_{\sigma}^{+}\right), \\
a_{I I}(\tilde{u}, \tilde{v})=-\left(\left(A_{2} \tilde{u}, \nabla \tilde{v}\right)\right)+\left(A_{1} \tilde{u}, \tilde{v}\right), \tilde{u}, \tilde{v} \in H^{1}\left(\Omega, \mathbb{C}_{\sigma}^{+}\right) .
\end{gathered}
$$

Analogously, we associate with the sesquilinear $b(.,$.$) defined by (22)$ the sesquilinear forms $b_{I}(.,$.$) and b_{I I}(.,$.

$$
\begin{gathered}
b_{I}(\tilde{u}, \tilde{v})=\left(\left(B_{22} \nabla \tilde{u}, \nabla \tilde{v}\right)\right), \tilde{u}, \tilde{v} \in H^{1}\left(\Omega, \mathbb{C}_{\sigma}^{+}\right), \\
b_{I I}(\tilde{u}, \tilde{v})=-\left(\left(B_{2} \tilde{u}, \nabla \tilde{v}\right)\right)+\left(B_{1} \tilde{u}, \tilde{v}\right), \tilde{u}, \tilde{v} \in H^{1}\left(\Omega, \mathbb{C}_{\sigma}^{+}\right) .
\end{gathered}
$$

We remark that, for $\tilde{u} \in H^{1}\left(\Omega, \mathbb{C}_{\sigma}^{+}\right)$,

$$
a_{I}(\tilde{u}, \tilde{u}) \in \mathbb{R}, \quad b_{I}(\tilde{u}, \tilde{u}) \in \mathbb{R} .
$$


Lemma 1. Let $a_{e}$ and $C_{b}$ be positive constants such that

$$
\begin{gathered}
a_{I}(\tilde{u}, \tilde{u}) \geq a_{e}|\tilde{u}|_{H^{1}\left(\Omega, \mathbb{C}_{\sigma}^{+}\right)}^{2}, \tilde{u} \in H^{1}\left(\Omega, \mathbb{C}_{\sigma}^{+}\right), \\
\left|b_{I}(\tilde{u}, \tilde{u})\right| \leq C_{b}|\tilde{u}|_{H^{1}\left(\Omega, \mathbb{C}_{\sigma}^{+}\right)}^{2}, \tilde{u} \in H^{1}\left(\Omega, \mathbb{C}_{\sigma}^{+}\right),
\end{gathered}
$$

If Rep $\geq \sigma>0$ and $|\bar{p} \tilde{k}| \leq \tilde{C}$, then there exists a positive constant $e(p)$ such that (25) holds.

Proof: From (32) and (33) we find

$$
\operatorname{Re} a_{p}(\tilde{u}, \tilde{u}) \geq\|\tilde{u}\|_{L^{2}\left(\Omega, \mathbb{C}_{\sigma}^{+}\right)}^{2}+\frac{1}{|p|^{2}}\left(a_{e} \operatorname{Re} p-C_{b} \tilde{C}\right)|\tilde{u}|_{H^{1}\left(\Omega, \mathbb{C}_{\sigma}^{+}\right)}^{2} .
$$

To have (25) it is sufficient to choose $\sigma$ such that

$$
\sigma>\frac{C_{b} \tilde{C}}{a_{e}}
$$

As an example we notice that, for the kernel $k(t)=\frac{1}{\tau} e^{-\frac{t}{\tau}}, \tau>0$, which was introduced in equation (8), we have $\bar{p} \tilde{k}=\frac{\bar{p}}{1+\tau p}$ and then $|\bar{p} \tilde{k}| \leq \frac{1}{\tau}$ for $\operatorname{Re} p \geq 0$.

For more general sesquilinear forms $a_{p}(.,$.$) we have the following sufficient$ conditions:

Lemma 2. Let us suppose that $a_{I}(.,$.$) defined by (28) satisfies (32). If$

$$
|b(\tilde{u}, \tilde{u})| \leq C_{b}\|\tilde{u}\|_{H^{1}\left(\Omega, \mathbb{C}_{\sigma}^{+}\right)}^{2}, \tilde{u} \in H^{1}\left(\Omega, \mathbb{C}_{\sigma}^{+}\right),
$$

and

$$
|\tilde{k}|=O\left(|p|^{-1}\right)
$$

then there exists $\sigma>0$ such that for $p \in \mathbb{C}_{\sigma}^{+}, a_{p}(.,$.$) defined by (20) satisfies$ (25).

Proof: Using convenient algebraic manipulations, we can show that

$$
\left|\frac{1}{p} a_{I I}(\tilde{u}, \tilde{u})\right| \leq \frac{1}{|p|^{2}} \frac{1}{4 \epsilon^{2}}\left\|A_{2}\right\|_{\infty}^{2}|\tilde{u}|_{H^{1}\left(\Omega, \mathbb{C}_{\sigma}^{+}\right)}^{2}+\left(2 \epsilon^{2}+\frac{1}{|p|}\left\|A_{1}\right\|_{\infty}\right)\|\tilde{u}\|_{L^{2}\left(\Omega, \mathbb{C}_{\sigma}^{+}\right)}^{2},
$$

$\forall \tilde{u} \in H^{1}\left(\Omega, \mathbb{C}_{\sigma}^{+}\right)$, for all $\epsilon \neq 0$. From (28) and (35) we get the estimate

$$
\operatorname{Re} a_{p}(\tilde{u}, \tilde{u}) \geq e_{1}(p)|\tilde{u}|_{H^{1}\left(\Omega, \mathbb{C}_{\sigma}^{+}\right)}^{2}+e_{0}(p)\|\tilde{u}\|_{L^{2}\left(\Omega, \mathbb{C}_{\sigma}^{+}\right)}^{2}, \forall \tilde{u} \in H_{0}^{1}\left(\Omega, \mathbb{C}_{\sigma}^{+}\right),
$$

with $e_{1}(p)$ and $e_{0}(p)$ defined respectively by

$$
e_{1}(p)=\frac{1}{|p|^{2}}\left(a_{e} \operatorname{Re} p-\frac{1}{4 \epsilon^{2}}\left\|A_{2}\right\|_{\infty}^{2}-C_{b}|\tilde{k}||p|\right)
$$

and

$$
e_{0}(p)=1-2 \epsilon^{2}-\frac{1}{|p|}\left\|A_{1}\right\|_{\infty}-C_{b} \frac{|\tilde{k}|}{|p|} .
$$

Now we use (36) and we conclude that there exists $\epsilon \neq 0$ and $\sigma \in \mathbb{R}^{+}$such that $e_{1}(p)$ and $e_{0}(p)$ satisfy

$$
e_{i}(p)>0, \forall p \in \mathbb{C}_{\sigma}^{+}, i=0,1 .
$$


Lemma 3. Let us suppose that $a_{I}(.,$.$) defined by (28) satisfies (32). If the$ sesquilinear form $b_{I}(.,$.$) satisfies$

$$
\begin{gathered}
b_{I}(\tilde{u}, \tilde{u}) \geq b_{e}|\tilde{u}|_{H^{1}\left(\Omega, \mathbb{C}_{\sigma}^{+}\right)}^{2}, \tilde{u} \in H^{1}\left(\Omega, \mathbb{C}_{\sigma}^{+}\right), \\
\operatorname{Re} \frac{\tilde{k}}{p}>0
\end{gathered}
$$

and

$$
|\tilde{k}|=O(1),
$$

then there exists $\sigma>0$ such that for $p \in \mathbb{C}_{\sigma}^{+}, a_{p}(.,$.$) defined by (20) satisfies$ (25).

Proof: Let us suppose now that the sesquilinear form $b_{I}(.,$.$) satisfies (42).$ For $b_{I I}(.,$.$) we can prove that$

$$
\left|\frac{1}{p} \tilde{k} b_{I I}(\tilde{u}, \tilde{u})\right| \leq \frac{|\tilde{k}|^{2}}{|p|^{2}} \frac{1}{4 \eta^{2}}\left\|B_{2}\right\|_{\infty}^{2}|\tilde{u}|_{H^{1}\left(\Omega, \mathbb{C}_{\sigma}^{+}\right)}^{2}+\left(2 \eta^{2}+\frac{|\tilde{k}|}{|p|}\left\|B_{1}\right\|_{\infty}\right)\|\tilde{u}\|_{L^{2}\left(\Omega, \mathbb{C}_{\sigma}^{+}\right)}^{2},
$$

$\forall \tilde{u} \in H^{1}\left(\Omega, \mathbb{C}_{\sigma}^{+}\right)$, for $\eta \neq 0$. As (43) holds, from (42) and (45), we conclude that

$$
\operatorname{Re} a_{p}(\tilde{u}, \tilde{u}) \geq e_{1}(p)|\tilde{u}|_{H^{1}\left(\Omega, \mathbb{C}_{\sigma}^{+}\right)}^{2}+e_{0}(p)\|\tilde{u}\|_{L^{2}\left(\Omega, \mathbb{C}_{\sigma}^{+}\right)}^{2}, \forall \tilde{u} \in H_{0}^{1}\left(\Omega, \mathbb{C}_{\sigma}^{+}\right),
$$

with $e_{1}(p)$ and $e_{0}(p)$ defined respectively by

$$
e_{1}(p)=\frac{1}{|p|^{2}}\left(a_{e} \operatorname{Re} p+b_{e} \operatorname{Re}(\tilde{k} \bar{p})-\frac{1}{4 \epsilon^{2}}\left\|A_{2}\right\|_{\infty}^{2}-\frac{1}{4 \eta^{2}}|\tilde{k}|^{2}\left\|B_{2}\right\|_{\infty}^{2}\right)
$$

and

$$
e_{0}(p)=1-2 \epsilon^{2}-2 \eta^{2}-\frac{1}{|p|}\left\|A_{1}\right\|_{\infty}-\frac{|\tilde{k}|}{|p|}\left\|B_{1}\right\|_{\infty},
$$

for all $\epsilon, \eta \neq 0$. Using now condition (44) we guarantee that there exist $\epsilon, \eta \neq 0$ and $\sigma \in \mathbb{R}^{+}$such that $e_{1}(p)$ and $e_{0}(p)$ satisfy (41).

It is clear that if $a_{i}=b_{i}=0, i=1,2, A_{1}=B_{1}=0$ and $A_{22}, B_{22}$ are diagonal matrices such that

$$
a_{i i} \geq \alpha_{e}>0 \text { in } \bar{\Omega},
$$

and

$$
b_{i i} \geq \beta_{e}>0 \text { in } \bar{\Omega},
$$

then (32), (42) and (35), respectively, hold with $a_{e}=\alpha_{e}, b_{e}=\beta_{e}$ and $C_{b}=$ $\left\|B_{22}\right\|_{\infty}$.

If we consider the kernel $k(t)=\frac{1}{\tau} e^{-\frac{t}{\tau}}, \tau>0$, introduced in (8), then $\tilde{k}=\frac{1}{1+\tau p}$ satisfies (36). 


\section{Discretization in the Laplace space}

\subsection{The finite element solution}

In order to simplify the presentation, in what follows we consider homogeneous Dirichlet boundary conditions, that is, $\psi=0$. By $C^{0}\left(\bar{\Omega}, \mathbb{C}_{\sigma}^{+}\right)$we represent the space of functions $\tilde{u}: \bar{\Omega} \times \mathbb{C}_{\sigma}^{+} \rightarrow \mathbb{C}$ depending on $x, p$, continuous in $\bar{\Omega}$ and analytic in $\mathbb{C}_{\sigma}^{+}$.

By $P_{m}(x, p)$ we denote a polynomial in space variables of degree $\leq m$ with coefficients depending on $p$ analytic in $\mathbb{C}_{\sigma}^{+}$. We consider a sequence of triangulations $\mathcal{T}_{H}$, with diameter $H=\max _{\Delta \in \mathcal{T}_{H}} \operatorname{diam}(\Delta)$, obtained by regular refinement (see [31]). By $\Lambda$ we denote the sequence of diameters of the sequence of triangulations. Let $V_{H, m}\left(\mathbb{C}_{\sigma}^{+}\right), H \in \Lambda$, be the corresponding sequence of finite element spaces:

$$
\begin{aligned}
V_{H, m}\left(\mathbb{C}_{\sigma}^{+}\right)= & \left\{\tilde{u} \in C^{0}\left(\bar{\Omega}, \mathbb{C}_{\sigma}^{+}\right): \tilde{u}=0 \text { on } \partial \Omega,\right. \\
& \left.\tilde{u}(x, p)=P_{m}(x, p), x \in \Delta, \Delta \in \mathcal{T}_{H}, p \in \mathbb{C}_{\sigma}^{+}\right\} .
\end{aligned}
$$

We denote by $V_{H, m}\left(\mathbb{C}_{\sigma}^{+}\right)^{\prime}$ the dual space of $V_{H, m}\left(\mathbb{C}_{\sigma}^{+}\right)$.

We remark that $V_{H, m}\left(\mathbb{C}_{\sigma}^{+}\right) \subset H_{0}^{1}\left(\Omega, \mathbb{C}_{\sigma}^{+}\right)$. Let $\left\{\phi, i=1, \ldots, n_{H}\right\}$ be a finite element basis of $V_{H, m}\left(\mathbb{C}_{\sigma}^{+}\right)$, where $\phi_{i}$ depends only on $x$. The Ritz-Galerkin approximation for the solution of $(24)$ is a function $\tilde{c}_{H} \in V_{H, m}\left(\mathbb{C}_{\sigma}^{+}\right)$such that

$$
a_{p}\left(\tilde{c}_{H}, \tilde{v}_{H}\right)=\ell_{H}\left(\tilde{v}_{H}\right), \forall \tilde{v}_{H} \in V_{H, m}\left(\mathbb{C}_{\sigma}^{+}\right),
$$

where $a_{p}(.,$.$) is defined by (20)$ and $\ell_{H}: V_{H, m}\left(\mathbb{C}_{\sigma}^{+}\right) \rightarrow \mathbb{C}$,

$$
\ell_{H}\left(\tilde{v}_{H}\right)=\ell\left(\tilde{v}_{H}\right), \tilde{v}_{H} \in V_{H, m}\left(\mathbb{C}_{\sigma}^{+}\right),
$$

with $\ell$ defined by $(23)$.

The existence and uniqueness of the previous finite element solution is consequence of the ellipticity of the bilinear form $a_{p}(.,$.$) .$

Theorem 2. If $f \in L^{2}\left(\mathbb{R}^{+}, L^{2}(\Omega)\right), c_{0} \in L^{2}(\Omega)$ and under the assumption of Theorem 1 , there exists a positive $\sigma$ such that, for each $p \in \mathbb{C}_{\sigma}^{+}$, the problem (50) has a unique solution $\tilde{c}_{H} \in V_{H, m}\left(\mathbb{C}_{\sigma}^{+}\right)$.

The finite element solution $\tilde{c}_{H} \in V_{H, m}\left(\mathbb{C}_{\sigma}^{+}\right), \tilde{c}_{H}=\sum_{i=1}^{n_{H}} a_{i} \phi_{i}$, where $a_{i}$ depends on $p$, is obtained solving the linear system

$$
\left[a_{p}\left(\phi_{i}, \phi_{j}\right)\right]\left[a_{i}\right]=\left[\ell\left(\phi_{j}\right)\right] .
$$

We remark that the variational equation (50) is equivalent to the following problem: find $\tilde{c}_{H} \in V_{H, m}\left(\mathbb{C}_{\sigma}^{+}\right)$such that

$$
L_{H} \tilde{c}_{H}=\ell_{H} \text { in } V_{H, m}\left(\mathbb{C}_{\sigma}^{+}\right),
$$

with $L_{H}: V_{H, m}\left(\mathbb{C}_{\sigma}^{+}\right) \rightarrow V_{H, m}\left(\mathbb{C}_{\sigma}^{+}\right)^{\prime}$, defined by

$$
L_{H} \tilde{u}_{H}\left(\tilde{v}_{H}\right)=a_{p}\left(\tilde{u}_{H}, \tilde{v}_{H}\right), \tilde{u}_{H}, \tilde{v}_{H} \in V_{H, m}\left(\mathbb{C}_{\sigma}^{+}\right) .
$$

Theorem 2 establishes a sufficient condition for the existence of a unique solution of the equation (52), $\tilde{c}_{H}=L_{H}^{-1} \ell_{H}$. 


\subsection{Error estimates for the finite element solution}

Let $\Pi_{H}: H_{0}^{1}\left(\Omega, \mathbb{C}_{\sigma}^{+}\right) \rightarrow V_{H, m}\left(\mathbb{C}_{\sigma}^{+}\right)$be the finite element projection operator. Under the assumption of Theorem 1 , there exists a unique solution $\tilde{c} \in$ $H_{0}^{1}\left(\Omega, \mathbb{C}_{\sigma}^{+}\right)$of $(24)$ and a unique solution $\tilde{c}_{H} \in V_{H, m}\left(\mathbb{C}_{\sigma}^{+}\right)$of $(50)$. As $H_{0}^{1}\left(\Omega, \mathbb{C}_{\sigma}^{+}\right)$ and $V_{H, m}\left(\mathbb{C}_{\sigma}^{+}\right)$are Hilbert spaces we consider $\Pi_{H}: H_{0}^{1}\left(\Omega, \mathbb{C}_{\sigma}^{+}\right)^{\prime} \rightarrow V_{H, m}\left(\mathbb{C}_{\sigma}^{+}\right)^{\prime}$. Let $S_{H}: H_{0}^{1}\left(\Omega, \mathbb{C}_{\sigma}^{+}\right) \rightarrow V_{H, m}$ be defined by

$$
S_{H}=L_{H}^{-1} \Pi_{H} L
$$

This operator satisfies

$$
S_{H}=I_{d} \text { in } V_{H, m} .
$$

In the next theorem we establish the error estimate for $\tilde{c}_{H}$.

Theorem 3. Let us suppose that the finite element spaces $V_{H, m}\left(\mathbb{C}_{\sigma}^{+}\right)$, for $H \in$ $\Lambda$, are constructed using a sequence of triangulations $\mathcal{T}_{H}$, with diameter $H \in \Lambda$, obtained by regular refinement. Under the assumption of Theorem 1 , there exists a unique solution $\tilde{c} \in H_{0}^{1}\left(\Omega, \mathbb{C}_{\sigma}^{+}\right)$of (24), a unique solution $\tilde{c}_{H} \in V_{H, m}\left(\mathbb{C}_{\sigma}^{+}\right)$of (50) and a positive constant $C$, independent of $\tilde{c}, H$ and $p$, such that, for $H \in \Lambda$ small enough, we have

$$
\left\|\tilde{c}-\tilde{c}_{H}\right\|_{H^{1}\left(\Omega, \mathbb{C}_{\sigma}^{+}\right)} \leq C H^{m}\|\tilde{c}\|_{H^{m+1}\left(\Omega, \mathbb{C}_{\sigma}^{+}\right)}
$$

provided that $\tilde{c} \in H^{m+1}\left(\Omega, \mathbb{C}_{\sigma}^{+}\right)$.

Proof: Following the proof of Theorems 3 of [9] we start by proving that

$$
\left\|\tilde{c}-\tilde{c}_{H}\right\|_{H^{1}\left(\Omega, \mathbb{C}_{\sigma}^{+}\right)} \leq C\left\|\tilde{c}-\tilde{v}_{H}\right\|_{H^{1}\left(\Omega, \mathbb{C}_{\sigma}^{+}\right)}, \forall \tilde{v}_{H} \in V_{H, m}\left(\mathbb{C}_{\sigma}^{+}\right) .
$$

As by using (53) we have

$$
\tilde{v}_{H}=S_{H} \tilde{v}_{H}
$$

then

$$
\begin{aligned}
\left\|\tilde{c}-\tilde{c}_{H}\right\|_{H^{1}\left(\Omega, \mathbb{C}_{\sigma}^{+}\right)} & \leq\left\|\tilde{c}-\tilde{v}_{H}\right\|_{H^{1}\left(\Omega, \mathbb{C}_{\sigma}^{+}\right)}+\left\|\tilde{c}_{H}-\tilde{v}_{H}\right\|_{H^{1}\left(\Omega, \mathbb{C}_{\sigma}^{+}\right)} \\
& =\left\|\tilde{c}-\tilde{v}_{H}\right\|_{H^{1}\left(\Omega, \mathbb{C}_{\sigma}^{+}\right)}+\left\|S_{H}\left(\tilde{c}-\tilde{v}_{H}\right)\right\|_{H^{1}\left(\Omega, \mathbb{C}_{\sigma}^{+}\right)}
\end{aligned}
$$

and we conclude that

$$
\left\|\tilde{c}-\tilde{c}_{H}\right\|_{H^{1}\left(\Omega, \mathbb{C}_{\sigma}^{+}\right)} \leq\left(1+\left\|S_{H}\right\|\right)\left\|\tilde{c}-\tilde{v}_{H}\right\|_{H^{1}\left(\Omega, \mathbb{C}_{\sigma}^{+}\right)},
$$

for $\tilde{v}_{H} \in V_{H, m}\left(\mathbb{C}_{\sigma}^{+}\right)$, where $\left\|S_{H}\right\|$, for $H \in \Lambda$ with $H$ small enough, has a bound independent of $p \in \mathbb{C}_{\sigma}^{+}$.

Moreover Theorem 4 of [9] allows us to conclude that there exists a positive constant $C$ independent on $\tilde{c}, H$ and $p$ such that, for $H$ small enough, we have

$$
\left\|\tilde{c}-\Pi_{H} \tilde{c}\right\|_{H^{1}\left(\Omega, \mathbb{C}_{\sigma}^{+}\right)} \leq C H^{m}\|\tilde{c}\|_{H^{m+1}\left(\Omega, \mathbb{C}_{\sigma}^{+}\right)} .
$$

From (55) and (56) we finally obtain (54). 


\section{Returning to the initial variables}

To return to the initial variables we need to apply the Laplace inverse to both members of an inequality of type (56) with convenient norms.

An essential tool to recover the initial variables is the Paley-Wiener Theorem. To present such lemma we introduce the space $L^{2}\left(\mathbb{R}^{+}, H^{1}(\Omega), \sigma\right)$ as the space of functions $v: \mathbb{R}^{+} \rightarrow H^{1}(\Omega)$ such that

$$
\|v\|_{L^{2}\left(\mathbb{R}^{+}, H^{1}(\Omega), \sigma\right)}=\left(\int_{\mathbb{R}^{+}} e^{-2 \sigma t}\|v(t)\|_{H^{1}(\Omega)}^{2} d t\right)^{1 / 2}
$$

is finite. In $L^{2}\left(\mathbb{R}^{+}, H^{1}(\Omega), \sigma\right)$ we consider the inner product

$$
(u, v)_{L^{2}\left(\mathbb{R}^{+}, H^{1}(\Omega), \sigma\right)}=\int_{\mathbb{R}^{+}}(u(t), v(t))_{H^{1}(\Omega)} e^{-2 \sigma t} d t, u, v \in L^{2}\left(\mathbb{R}^{+}, H^{1}(\Omega), \sigma\right)
$$

which induces the norm defined by (57). We also consider the Hardy space $\mathcal{H}^{2}\left(\mathbb{C}_{\sigma}^{+}, H^{m+1}(\Omega)\right)$ of holomorphic functions $\tilde{f}: \mathbb{C}_{\sigma}^{+} \rightarrow H^{m+1}(\Omega)$ such that

$$
\|\tilde{f}\|_{\mathcal{H}^{2}\left(\mathbb{C}_{\sigma}^{+}, H^{m+1}(\Omega)\right)}=\left(\sup _{p_{1}>\sigma} \int_{\mathbb{R}}\left\|\tilde{f}\left(p_{1}+i p_{2}\right)\right\|_{H^{m+1}(\Omega)}^{2} d p_{2}\right)^{1 / 2}<\infty .
$$

Lemma 4. [Paley-Wiener Theorem] The Laplace transform $\mathcal{L}: L^{2}\left(\mathbb{R}^{+}, H^{m+1}(\Omega), \sigma\right) \rightarrow \mathcal{H}^{2}\left(\mathbb{C}_{\sigma}^{+}, H^{m+1}(\Omega)\right)$ is an isometric isomorphism.

Inequality (54) allows us to write

$$
\left\|\tilde{c}-\tilde{c}_{H}\right\|_{\mathcal{H}^{2}\left(\mathbb{C}_{\sigma}^{+}, H^{1}(\Omega)\right)} \leq C H^{m}\|\tilde{c}\|_{\mathcal{H}^{2}\left(\mathbb{C}_{\sigma}^{+}, H^{m+1}(\Omega)\right)} .
$$

Applying Paley-Wiener Theorem we get the main result of this paper:

Theorem 4. Let us suppose that the finite element spaces $V_{H, m}\left(\mathbb{C}_{\sigma}^{+}\right)$, for $H \in$ $\Lambda$, are constructed using a sequence of triangulations $\mathcal{T}_{H}$, with diameter $H \in \Lambda$, obtained by regular refinement. Under the assumption of Theorem 1 there exist a unique solution $\tilde{c} \in H_{0}^{1}\left(\Omega, \mathbb{C}_{\sigma}^{+}\right)$of (24), a unique solution $\tilde{c}_{H} \in V_{H, m}\left(\mathbb{C}_{\sigma}^{+}\right)$of (50) and a positive constant $C$, independent of $\tilde{c}, H$ and $p$, such that, for $H \in \Lambda$ small enough, $c=\mathcal{L}^{-1} \tilde{c}, c_{H}=\mathcal{L}^{-1} \tilde{c}_{H}$ satisfy

$$
\left\|c-c_{H}\right\|_{L^{2}\left(\mathbb{R}^{+}, H^{1}(\Omega), \sigma\right)} \leq C H^{m}\|c\|_{L^{2}\left(\mathbb{R}^{+}, H^{m+1}(\Omega), \sigma\right)},
$$

provided that $\tilde{c} \in L^{2}\left(\mathbb{R}^{+}, H^{m+1}(\Omega), \sigma\right)$.

\section{Numerical simulation}

In this section we give one example of application of the method based on the Laplace transform described in Section 4 combined with the algorithm developed in [1] for the inverse Laplace transform.

We consider the integro-differential equation (1) with $\Omega=(0,1) \times(0,1), A=$ $B=-\Delta$, where $\Delta$ denotes the Laplace operator and $k(s)=\frac{1}{\tau} e^{-\frac{s}{\tau}}, \tau=0.01$. 


\begin{tabular}{|c|c|c||c|c|}
\hline & \multicolumn{2}{|c||}{ linear elements } & \multicolumn{2}{c|}{ quadratic elements } \\
\hline$N$ & Error & Rate & Error & Rate \\
\hline 10 & 0.000173033 & 1.01 & $9.57556 \mathrm{e}-006$ & 1.99 \\
20 & $8.61843 \mathrm{e}-005$ & 1.00 & $2.40258 \mathrm{e}-006$ & 2.00 \\
30 & $5.74128 \mathrm{e}-005$ & 1.00 & $1.06856 \mathrm{e}-006$ & 2.00 \\
40 & $4.30481 \mathrm{e}-005$ & 1.00 & $6.01213 \mathrm{e}-007$ & 2.00 \\
50 & $3.44342 \mathrm{e}-005$ & 1.00 & $3.84821 \mathrm{e}-007$ & 2.00 \\
60 & $2.86932 \mathrm{e}-005$ & 1.00 & $2.67253 \mathrm{e}-007$ & 2.00 \\
70 & $2.45932 \mathrm{e}-005$ & 1.00 & $1.96357 \mathrm{e}-007$ & 2.00 \\
80 & $2.15184 \mathrm{e}-005$ & - & $1.50339 \mathrm{e}-007$ & - \\
\hline
\end{tabular}

Table 1: Errors and rates obtained for linear and quadratic elements computed with the norm $\|\cdot\|_{H^{1}\left(\Omega, \mathbb{C}_{\sigma}^{+}\right)}$.

The function $f$, the initial and boundary conditions are such that the IBVP has the following solution

$$
c(x, t)=\cos (t) x_{1} x_{2}\left(1-x_{1}\right)\left(1-x_{2}\right),\left(x_{1}, x_{2}\right) \in \bar{\Omega}, t \in \mathbb{R}_{0}^{+} .
$$

In $\Omega$ we introduce a triangulation $\mathcal{T}_{H}$ induced by a uniform rectangular grid defined considering, in $[0,1] \times[0,1],(N+1) \times(N+1)$ equally spaced points.

First we illustrate the convergence result of Theorem 3 by comparing the numerical and the exact solution in the Laplace space. Let $\tilde{c}$ be the solution of (15), that is,

$$
\tilde{c}(x)=\frac{p}{p^{2}+1} x_{1} x_{2}\left(1-x_{1}\right)\left(1-x_{2}\right),\left(x_{1}, x_{2}\right) \in \bar{\Omega},
$$

and $\tilde{c}_{H} \in V_{H, m}\left(\mathbb{C}_{\sigma}^{+}\right)$be the solution of $(50)$, where $\sigma=100$ and $p=100+100 i$. We consider $m=1,2$, that is, we use linear and quadratic finite elements.

Assuming that $\left\|\tilde{c}-\tilde{c}_{H}\right\|_{H^{1}\left(\Omega, \mathbb{C}_{\sigma}^{+}\right)} \simeq C H^{q}$, we show that $q \simeq m$. The convergence rate is computed using the formula

$$
\text { Rate }=\frac{\ln \left(\frac{\left\|\tilde{c}-\tilde{c}_{H_{1}}\right\|_{H^{1}\left(\Omega, \mathbb{C}_{\sigma}^{+}\right)}}{\left\|\tilde{c}-\tilde{c}_{H_{2}}\right\|_{H^{1}\left(\Omega, \mathbb{C}_{\sigma}^{+}\right)}^{+}}\right)}{\ln \left(\frac{H_{1}}{H_{2}}\right)},
$$

where $H_{1}$ and $H_{2}$ are the diameters of two consecutive triangulations. We show the numerical results in Table 1. In Figure 1, the logarithm of the norm of the error, $\ln \left(\left\|\tilde{c}-\tilde{c}_{H}\right\|_{H^{1}\left(\Omega, \mathbb{C}_{\sigma}^{+}\right)}\right)$, is plotted versus the logarithm of the mesh size. The straight lines are the least-squares fit to the points and have slopes 1.0022 and 1.9979 for linear and quadratic elements, respectively. The experiments show that the convergence rates are, approximately, 1 and 2 when linear and quadratic elements are used, respectively, agreeing with the estimate of Theorem 3 .

Let us now illustrate the convergence behavior of the numerical solution in the initial variables. For each time $t$, the Laplace inverse of the finite element solution is computed using the algorithm developed in [1] with the following 


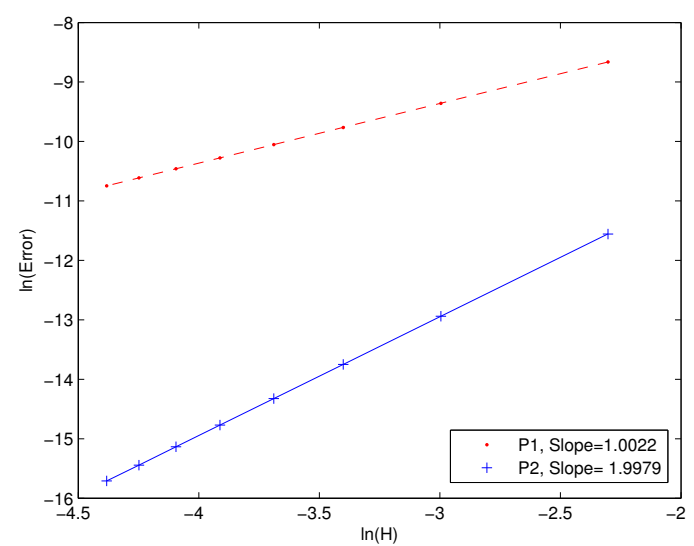

Figure 1: $\ln \left(\left\|\tilde{c}-\tilde{c}_{H}\right\|_{H^{1}\left(\Omega, \mathbb{C}_{\sigma}^{+}\right)}\right)$versus $\ln (H)$.

parameters, according the notation used in the mentioned paper: $\alpha=0, T=$ $0.8 t, E_{R}=10^{-8}, \gamma=-\frac{\ln \left(E_{R}\right)}{1.6 T}, M=50$ and $T o l=\frac{T}{e^{2 \gamma T} N^{2}}$.

We remark that we observe the bound

$$
\left\|c(t)-c_{H}(t)\right\|_{H^{1}(\Omega)} \leq C H^{m},
$$

which is a stronger estimate when compared with the result in Theorem 4,

$$
\int_{\mathbb{R}^{+}} e^{-2 \sigma t}\left\|c(t)-c_{H}(t)\right\|_{H^{1}(\Omega)}^{2} d t \leq C H^{2 m}\|c\|_{L^{2}\left(\mathbb{R}^{+}, H^{m+1}(\Omega), \sigma\right)}^{2} .
$$

In Table 2 we present the numerical error $\operatorname{Error}(t)=\left\|c(t)-c_{H}(t)\right\|_{H^{1}(\Omega)}$ and Rate $(t)$,

$$
\operatorname{Rate}(t)=\frac{\ln \left(\frac{\left\|c(t)-c_{H_{1}}(t)\right\|_{H^{1}(\Omega)}}{\left\|c(t)-c_{H_{2}}(t)\right\|_{H^{1}(\Omega)}}\right)}{\ln \left(\frac{H_{1}}{H_{2}}\right)},
$$

where $H_{1}$ and $H_{2}$ are the diameters of two consecutive triangulations, for $t=0.1$, $t=1$ and $t=10$ computed using linear elements. The numerical results show that the convergence rate is 1 when linear elements are used.

The numerical errors Error $(t)$ and Rate $(t)$ for $t=0.1, t=1$ and $t=10$, for quadratic elements, are presented in Table 3 . The numerical results show that the convergence rate is about 2 when quadratic elements are used.

Figure 2, plots the data from both tables 2 and 3 and illustrates the bounds established by Theorem 4 .

When $N$ increases we observe a deterioration of the convergence rates. This behavior was expected since, for large values of $N$, the error of the spatial discretization is very small and the error $\left\|c(t)-c_{H}(t)\right\|_{H^{1}(\Omega)}$ is dominated by the error induced by numerical Laplace inversion.

Finally we present some results obtained considering the $L^{2}$ norm in the measurement of the error. In Table 4 we present the results obtained with 


\begin{tabular}{|c|c|c|c|c|c|c|}
\hline$N$ & Error(0.1) & Rate(0.1) & Error(1) & Rate(1) & Error(10) & Rate(10) \\
\hline 10 & 0.0241023 & 1.00 & 0.0130785 & 0.99 & 0.0203103 & 0.99 \\
20 & 0.0120958 & 1.00 & 0.00656704 & 1.00 & 0.0101984 & 1.00 \\
30 & 0.00806943 & 1.00 & 0.00438147 & 1.00 & 0.00680427 & 1.00 \\
40 & 0.00605353 & 1.00 & 0.00328701 & 1.00 & 0.00510461 & 1.00 \\
50 & 0.00484337 & 1.00 & 0.00262995 & 1.00 & 0.00408422 & 1.00 \\
60 & 0.00403639 & 1.00 & 0.00219179 & 1.00 & 0.00340375 & 1.00 \\
70 & 0.00345989 & 1.00 & 0.00187876 & 1.00 & 0.00291763 & 1.00 \\
80 & 0.00302749 & - & 0.00164398 & - & 0.00255301 & - \\
\hline
\end{tabular}

Table 2: Errors and rates obtained for linear elements at $t=0.1,1,10$, computed with the norm $\|\cdot\|_{H^{1}}$.

\begin{tabular}{|c|c|c|c|c|c|c|}
\hline$N$ & Error(0.1) & Rate(0.1) & Error(1) & Rate(1) & Error(10) & Rate(10) \\
\hline 10 & 0.00134738 & 1.99 & 0.000731614 & 1.99 & 0.00113616 & 1.99 \\
20 & 0.000338144 & 1.99 & 0.000183679 & 1.98 & 0.00028516 & 1.99 \\
30 & 0.000150626 & 1.97 & $8.21509 \mathrm{e}-05$ & 1.89 & 0.000127113 & 1.97 \\
40 & $8.54939 \mathrm{e}-005$ & 1.87 & $4.76761 \mathrm{e}-05$ & 1.63 & $7.21529 \mathrm{e}-05$ & 1.84 \\
50 & $5.63455 \mathrm{e}-005$ & 1.61 & $3.31572 \mathrm{e}-05$ & 1.01 & $4.78133 \mathrm{e}-05$ & 1.49 \\
60 & $4.20235 \mathrm{e}-005$ & - & $2.75704 \mathrm{e}-05$ & - & $3.64293 \mathrm{e}-05$ & - \\
\hline
\end{tabular}

Table 3: Errors and rates obtained for quadratic elements at $t=0.1,1,10$, computed with the norm $\|\cdot\|_{H^{1}}$.

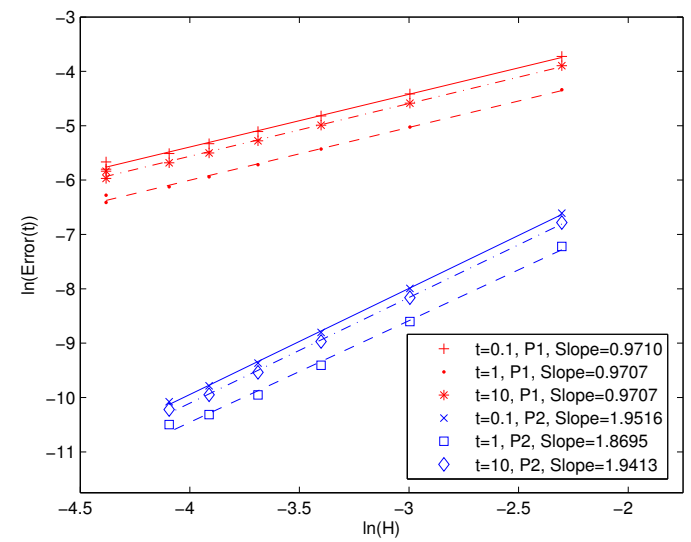

Figure 2: $\ln \left(\left\|c(t)-c_{H}(t)\right\|_{H^{1}(\Omega)}\right)$ versus $\ln (H)$.

linear elements that show a second order convergence rate. The errors and rates obtained with quadratic elements are presented in Table 5. These results show a third order convergence rate. However, when $N$ increases we observe, as before, a deterioration of this rate because the error $\left\|c(t)-c_{H}(t)\right\|_{L^{2}(\Omega)}$ is dominated by the error of the numerical Laplace inversion.

Also, we can only expect that the numerical Laplace inverse is computed with a high degree of accuracy for moderate values of $t$. In fact, for the example considered in the experiments, when we consider large values of $t$ (e.g. $t=100)$ 


\begin{tabular}{|c|c|c|c|c|c|c|}
\hline$N$ & Error(0.1) & Rate(0.1) & Error(1) & Rate(1) & Error(10) & Rate(10) \\
\hline 10 & 0.000923419 & 1.99 & 0.000516432 & 1.99 & 0.000788718 & 1.99 \\
20 & 0.000232886 & 2.00 & 0.000130441 & 2.00 & 0.000199163 & 2.00 \\
30 & 0.000103637 & 2.00 & $5.80592 \mathrm{e}-05$ & 2.00 & $8.86341 \mathrm{e}-05$ & 2.00 \\
40 & $5.83169 \mathrm{e}-005$ & 2.00 & $3.26734 \mathrm{e}-05$ & 2.00 & $4.98842 \mathrm{e}-05$ & 2.00 \\
50 & $3.7338 \mathrm{e}-005$ & 2.00 & $2.09267 \mathrm{e}-05$ & 2.00 & $3.19352 \mathrm{e}-05$ & 2.00 \\
60 & $2.59387 \mathrm{e}-005$ & 2.00 & $1.45307 \mathrm{e}-05$ & 2.00 & $2.21752 \mathrm{e}-05$ & 2.00 \\
70 & $1.90528 \mathrm{e}-005$ & 2.00 & $1.06773 \mathrm{e}-05$ & 2.00 & $1.62915 \mathrm{e}-05$ & 2.00 \\
80 & $1.45901 \mathrm{e}-005$ & - & $8.17607 \mathrm{e}-06$ & - & $1.24722 \mathrm{e}-05$ & - \\
\hline
\end{tabular}

Table 4: Errors and rates obtained for linear elements at $t=0.1,1,10$, computed with the norm $\|\cdot\|_{L^{2}}$.

\begin{tabular}{|c|c|c|c|c|c|c|}
\hline$N$ & Error(0.1) & Rate(0.1) & Error(1) & Rate(1) & Error(10) & Rate(10) \\
\hline 10 & $1.43765 \mathrm{e}-005$ & 3.00 & $7.81467 \mathrm{e}-06$ & 3.01 & $1.21519 \mathrm{e}-05$ & 3.01 \\
20 & $1.78621 \mathrm{e}-006$ & 2.99 & $9.69927 \mathrm{e}-07$ & 2.97 & $1.50822 \mathrm{e}-06$ & 2.99 \\
30 & $5.30795 \mathrm{e}-007$ & 2.91 & $2.91427 \mathrm{e}-07$ & 2.71 & $4.48644 \mathrm{e}-07$ & 2.92 \\
40 & $2.29777 \mathrm{e}-007$ & 2.64 & $1.33718 \mathrm{e}-07$ & 2.07 & $1.93656 \mathrm{e}-07$ & 2.55 \\
50 & $1.27406 \mathrm{e}-007$ & 2.17 & $8.41604 \mathrm{e}-08$ & 1.36 & $1.09575 \mathrm{e}-07$ & 1.94 \\
60 & $8.57563 \mathrm{e}-008$ & - & $6.57133 \mathrm{e}-08$ & - & $7.68772 \mathrm{e}-08$ & - \\
\hline
\end{tabular}

Table 5: Errors and rates obtained for quadratic elements at $t=0.1,1,10$, computed with the norm $\|\cdot\|_{L^{2}}$.

we don't observe a good agreement between the exact and numerical solution due to the limitations of the algorithm for the numerical Laplace inversion.

\section{Conclusions}

In this paper we consider a hybrid numerical method for the IBVP (1), (9), (10). The method is composed by three steps: in the first step, applying Laplace transforms, the given initial boundary value problem is replaced by an elliptic boundary value problem that depends on the Laplace parameter; in the second step the solution of this boundary value problem is approximated using the finite element method, for a choice of a finite set of quadrature points in the Laplace domain; finally, in the third stage, the numerical solution on the physical time space domain is obtained using numerical inverse Laplace transforms. The main result of this paper, Theorem 4, shows the theoretical error estimates for $c-c_{H}$. Although the norm used in the estimate (60) doens't give information for the error at a specific value of $t$, since it involves an integration over the time and a negative exponential in the variable $t$, the numerical results illustrate, for moderate values of $t$, that the method proposed has similar convergence behavior when compared to the results known for the standard finite element method for elliptic or parabolic problems.

\section{Acknowledgements}

The authors gratefully acknowledge the support of Centro de Matemática da Universidade de Coimbra (CMUC), funded by the European Regional De- 
velopment Fund through the program COMPETE and by the Portuguese Government through the FCT - Fundação para a Ciência e a Tecnologia under the project PEst-C/MAT/UI0324/2011 and the project UTAustin/MAT/0066/2008.

\section{References}

[1] J. Ahn, S. Kang, Y-H Kwon, A flexible inverse Laplace transform algorithm and its application, Computing 71 (2003) 115-131.

[2] A. Araújo, J.R. Branco, J.A. Ferreira, On the stability of a class of splitting methods for integro-differential equations, Applied Numerical Mathematics 59 (2009) 436-453.

[3] M. Aouadi, Hybrid Laplace transform-finite element method to a generalized electromagneto-thermoelastic problem, Applied Mathematical Modelling 31 (2007) 712-726.

[4] S. Barbeiro, J.A. Ferreira, Integro-differential models for percutaneous drug absorption, International Journal of Computer Mathematics 84 (2007) 451467.

[5] S. Barbeiro, J.A. Ferreira, Coupled vehicle-skin models for drug release, Computer Methods in Applied Mechanics and Engineering 198 (2009) 20782086.

[6] S. Barbeiro, J.A. Ferreira, L. Pinto, $H^{1}$-second order convergent estimates for non Fickian models, Applied Numerical Mathematics, 61 (2011) 201215 .

[7] N. Bauermeister, S. Shaw, Finite-element approximation of non-Fickian polymer diffusion, IMA Journal of Numerical Mathematics 30 (2010) 702730 .

[8] J.R. Branco, J.A. Ferreira, P. de Oliveira, Numerical methods for the generalized Fisher-Kolmogorov-Petrovskii-Piskunov equation, Applied Numerical Mathematics 57 (2007) 89-102.

[9] J. Brilla, Error analysis for Laplace transform finite element solution of hyperbolic equations, Numerische Mathematic 41 (1983) 55-62.

[10] K. Crump, Numerical inversion of Laplace transforms using a Fourier series approximation, Journal of the Association for Computing Machinery 2 (1976) 89-96.

[11] J.Cushman, X. Hu, F. Deng, Nonlocal reative transport with physical and chemical heterogeneity: localization error, Water Resources Research 31 (1995) 2219-2237.

[12] F. de Hoog, J. Knight, S. Stokes, An improved method for numerical inversion of Laplace transforms, SIAM Journal on Scientific and Statistical Computing 3 (1982) 357-366.

[13] H. Durbner, J. Abate, Numerical inversion of Laplace transforms by relating them to the finite Fourier cosines transforms, Journal of the Association for Computing Machinery 15 (1968) 115-123. 
[14] F. Durbin, Numerical inversion of Laplace transforms: an efficient improvement to Dubner and Abate's method, The Computer Journal 17 (1973) 371-376.

[15] A. El-Zein, J. Cater, D. Airey, Multiple-porosity contaminant transport by finite-element method, International Journal of Geomechanics 5 (2005) 24-34.

[16] R. Ewing, R. Lazarov, Y. Lin, Finite volume element approximations of nonlocal in time one-dimensional flows in porous media, Computing 64 (2000) 157-182.

[17] R. Ewing, R. Lazarov, Y. Lin, Finite volume element approximations of nonlocal reactive flows in porous media, Numerical Methods for Partial Differential Equations 16 (2000) 258-311.

[18] R. Ewing, Y. Lin, T. Sun, J. Wang, S. Zhang, Sharp $L^{2}$-error estimates and superconvergence of mixed finite element methods for non-Fickian flows in porous media, SIAM Journal of Numerical Analysis 40 (2002) 1538-1560.

[19] D. Farrel, D. Woodbury, E. Sudicky, Numerical modelling of mass transport in hydrogeologic environments: performance comparasion of the Laplace transform Galerkin and Arnoldi modal reduction schemes, Advances in Water Resources 21 (1998) 217-235.

[20] J.A. Ferreira, P. de Oliveira, Memory effects and random walks in reactiontransport systems, Applicable Analysis 86 (2007) 99-118.

[21] J.A. Ferreira, P. de Oliveira, Qualitative analysis of a delayed non Fickian model, Applicable Analysis 87 (2008) 873-886.

[22] A. Furman, S.P. Neuman, Laplace-transform analytic element solution of transient flow in porous media, Advances in Water Resources 26 (2003) 1229-1237.

[23] S.M. Hassahizadeh, On the transient non-Fickian dispersion theory, Transport in Porous Media 23 (1996) 107-124.

[24] D.D. Joseph, L. Preziosi, Heat waves, Reviews of Modern Physicsc 61 (1989) 41-73.

[25] Y. Lin, Semi-discrete finite element approximations for linear parabolic integro-differential equations with integrable kernels, Journal of Integral Equations and Applications 10 (1998) 51-83.

[26] Y. Lin, V. Thomée, L.B. Wahlbin, Ritz-Volterra projections to finiteelement spaces and applications to integrodifferential and related equations, SIAM Journal of Numerical Analysis 28(1991) 1047-1070.

[27] C. Maas, A hyperbolic dispersion equation to model the bounds of a contaminated groundwater body, Journal of Hydrology 226 (1999) 234-241.

[28] W. McLean, I.H. Sloan, V. Thomée, Time discretization via Laplace transformation of an integro-differential equation of parabolic type, Numerische Mathematik 10 (2006) 497-522. 
[29] S. Neuman, D. Tartakovski, Perspectives on theories of non-Fickian transport in heterogeneous medias, Advances in Water Resources 32 (2009) 678680 .

[30] S. Neuman, Y. Zhang, A quasi-linear theory of nonFickian and Fickian subsurgace dispersion I. Theoretical analysis with application to isotropic media, Advances in Water Resources 26 (1990) 887-902.

[31] J.T. Oden, J.N. Reddy, An Introduction to the Mathematical Theory of Finite Elements, New York, London, Sydney, Toronto,: John Willey and Sons, 1976.

[32] A.K. Pani, T.E. Peterson, 1996 Finite element methods with numerical quadrature for parabolic integro-differential equations, SIAM Journal of Numerical Analysis 33 (1996) 1084-1105.

[33] B. Rivière, S. Shaw, Discontinuous Galerkin finite element approximation of nonlinear non-Fickian diffusion in viscoelastic polymers, SIAM Journal of Numerical Analysis 44 (2006) 2245-2698.

[34] L. Ren, R. Zhang, Hybrid Laplace transform finite element method for solving the convection-dispersion problem, Advances in Water Resources, 23 (1999) 229-237.

[35] R. Sinha, R. Ewing, R. Lazarov, Some new error estimates of a semidiscrete finite volume method for a parabolic integro-differential equation with nonsmooth initial data, SIAM Journal of Numerical Analysis 43 (2006) 2320-2344.

[36] R. Sinha, R. Ewing, R. Lazarov, Mixed finite element method approximations of parabolic integro-differential equations with nonsmooth initial data, SIAM Journal of Numerical Analysis 47 (2009) 3269-3292.

[37] J. Sladek, S. Atluri, Meshless local Petrov-Galerkin method in anisotropic elasticity, Computer Modeling in Engineering and Science, 6 (2004) 477489.

[38] E. Sudicky, R. MacLaren, The Laplace transform Galerkin technique for large-scale simulation of mass transport in discretely fractured porous formations, Water Resources Research, 28 (1992) 499-514.

[39] D.M. Tartakovsky, S.P. Neuman, Transient flow in bounded randomly heterogeneous domains: 2. Localization of conditional mean equations and temporal nonlocality effects, Water Resources Research 34 (1998) 13-20.

[40] D.M. Tartakovsky, S.P. Neuman, Transient effective hydraulic conductivities under slowly and rapidly varying mean gradients in bounded threedimensional random media, Water Resources Research 34 (1998) 21-32.

[41] V. Thomée, N-Y. Zhang, Error estimates for semidiscrete finite element methods for parabolic integro-differential equations, Mathematics of Computation 53 (1989) 121-139. 
[42] V. Thomée, A high order parallel method for time discretization of parabolic type equations based on Laplace transformation and quadrature, Int. J. Numer. Anal. Model. 2 (2005) 86-96.

[43] M. Ye, S.P. Neuman, A. Guadagnini, D.M. Tartakovsky, Nonlocal and localized analyses of conditional mean transient flow in bounded, randomly heterogeneous porous media, Water Resources Research 40 (2004).

[44] N-Y Zhang, On fully discrete Galerkin approximation for partial integrodifferential equations of parabolic type, Mathematics of Computation 60 (1993) 133-166. 